\title{
Personalabbau und Ethik \\ Ein historischer Essay zur Frage, ob es richtig war, Kathryn McNeil zu entlassen
}

\author{
JÖRG MiCHAEL ROTHE
}

\section{Laying off Staff - Ethical Implications: An Historical Essay on the Issue of Laying off Kathryn McNeil}

Approximately 200 years ago, economic thinking changed fundamentally. The morality of the market emerged. However, it has been competing up to the present day with the older 'family' morality, which in turn can be traced back to Aristotle's concept of the 'oikes'. The laying off of Katbryn McNeil serves to exemplify this conflict of moralities. From an historical perspective, we might well live in a period of transition, in which the economy manages to reconcile the morality of the market with that of the family.

Keywords: Staff Lay off, Morality of the Market, Economy, Morality of the Family

\section{Die Moral der Familie und die Moral des Marktes}

Familien bauen kein Personal ab, wenn das Familieneinkommen abnimmt. Sie halten zusammen solange es geht. ${ }^{1}$ Hingegen wird in heutigen Unternehmen Personal abgebaut, um Gewinne zu erzielen oder um Verluste zu vermeiden, auch dann wenn die Existenz des Unternehmens nicht bedroht ist. Dies wird von den vom Personalabbau betroffenen Menschen oft als unmoralisch empfunden. ${ }^{2}$ Sie erleben sich nur noch als Mittel zum Zweck.

Vielleicht lässt sich diese Differenz besser verstehen, wenn man Moral nicht absolut denkt, sondern damit rechnet, dass eine ganz andere Moral gleichzeitig existiert und ähnliche Wirkungskraft entfalten kann. Ich treffe also eine Unterscheidung, und spre-

\footnotetext{
* $\quad$ Beitrag eingereicht am 22.05.2006; nach doppelt verdecktem Gutachterverfahren überarbeitete Fassung angenommen am 06.11.2006.

** Jörg Michael Rothe M.A., Beetstr. 12, D-32105 Bad Salzuflen, Tel.: +49-(0)5222-803331, E-Mail: jm.rothe@t-online.de, Forschungsschwerpunkte: Stadtgeschichte des Mittelalters und der Frühen Neuzeit, Sozial- und Wirtschaftsgeschichte.

1 Auch wenn Familien zunehmend selbst von ökonomischen Prinzipien beeinflusst werden und dadurch sich auch ihre Handlungsmuster verändern (vgl. Stierlin 1997: 147), lassen sich doch nach wie vor Unterschiede zwischen ihrer Moral und der des Marktes treffen.

2 Moral wird hier verstanden als ein in wechselseitigen Anerkennungsprozessen hervorgegangenes und als allgemein verbindlich ausgezeichnetes Handlungsmuster, welches normative Geltung hat (vgl. Pieper 2001: 21f.).
} 
che in diesem Aufsatz von einer Moral des Marktes und von einer Moral der Familie. ${ }^{3}$ Meine Hoffnung ist, dass - nach einer Zeit der ökonomischen Systemtransformation (vgl. Spree 2003: 27f.) - die unterschiedlichen Moralen zu einer auf einander bezogenen und sich gegenseitig respektierenden Gleichberechtigung finden.

Dirk Kurbjuweit berichtet in seinem Buch über McKinsey von einem Gespräch, das er mit dessen Chef, Jürgen Kluge, geführt hat:

„Jürgen Kluge habe ich bei unserer zweiten Begegnung vom McKinsey-Paradox erzählt: In fast jedem Einzelfall lassen sich gute Gründe für Effizienz und ökonomisches Verhalten finden, in der Summe kommt dabei eine Gesellschaft heraus, die nicht lebenswert ist. Kluge lächelte, wie er das oft tut, und sagte: ,Vielleicht haben Sie Recht.' Er erzählte von seiner Frau und dass er manchmal nach Hause komme und sie überschütte mit Erzählungen aus der McKinsey-Welt, gerade nach schwierigen Tagen und irgendwann sagt seine Frau, dass es noch etwas anderes gebe, eine Welt, die nicht McKinsey ist, und er solle jetzt McKinsey vergessen und in diese andere Welt eintauchen" (Kurbjuweit 2004: 179f.). ${ }^{4}$

Es gibt also zwei Welten. Eine davon ist draußen; dort wendet Jürgen Kluge die Spielregeln des Marktes an. Die andere Welt befindet sich drinnen im Haus; hier soll er zusammen mit seiner Frau eben diese Welt da draußen vergessen können; und dies auch deshalb, weil hier drinnen andere Spielregeln herrschen.

Unternehmensberater wie Jürgen Kluge haben draußen auf dem Markt schon dazu beigetragen, dass Mitarbeitende entlassen worden sind. Gleichwohl ist es nur schwer vorstellbar, dass sich ein Unternehmensberater oder Manager drinnen im Haus an den heimatlichen Küchentisch zu seiner fünfköpfigen Familie setzt und ihr ganz sachlich mitteilt, dass nun einem Familienmitglied gekündigt werden muss, da die Kosten mittlerweile höher als die Einnahmen seien.

Familien kennen keinen Personalabbau. Ein Vater entlässt seinen Sohn nicht. Eine Mutter kündigt der eigenen Tochter nicht.

In der Marktwirtschaft wird dagegen aus rein sachlichen Gründen Personal freigesetzt, sei es, dass Gewinne steigen sollen, dass Kosten gesenkt oder sei es, dass nur dadurch

Ich folge hier einer Unterscheidung, die Fritz B. Simon aus systemtheoretischer Überlegung zwischen Familie und Unternehmen vorgenommen hat (Simon 2005a). Den Herausgeberinnen und Herausgebern der zfwu bin ich für Kritik dankbar, die mich die Unterscheidung nunmehr zwischen Familie und Markt treffen lässt. Denn die von Simon herausgearbeiteten Unterschiede erklären mehr, wenn sie zwischen Familie und Markt konstruiert werden als zwischen Familien und Unternehmen. In den Unternehmen treffen die Moral der Familie und die Moral des Marktes aufeinander (vgl. Middelhoff 2004: 363). Die Spielregeln der Familie - wie Simon sie beschreibt - sind vielfach von Reziprozität geprägt, insbesondere durch die Langfristigkeit und die personale Gebundenheit der Austauschbeziehungen (vgl. dazu: Hollstein 2005).

$4 \quad$ Der Volkwirt und Wirtschaftshistoriker Carl-Ludwig Holtfrerich hat dieses von Dirk Kurbjuweit so genannte ,McKinsey-Paradox' in seiner kürzlich erschienenen „Streitschrift für mehr Arbeit“ als „Trugschluss der Verallgemeinerung“ (Holtfrerich 2007: 13) beschrieben und erklärt. „Was auf der Ebene einzelner Unternehmen oder Branchen funktioniert, wirkt auf der gesamtwirtschaftlichen Ebene nicht gleichermaßen. Diese Einsicht zu ignorieren, ist der Kardinalfehler der angebotsorientierten Wirtschaftspolitik, die sich von betriebswirtschaftlichem statt von volkswirtschaftlichem Denken leiten lässt..“ (ebd.: 138f.). 
das Überleben des Unternehmens gerettet werden kann. ${ }^{5}$ Personal wird freigesetzt, dass es sich hierbei um fühlende menschliche Wesen handelt, ist den folgenden Zitaten nicht zu entnehmen:

„Aus ökonomischer Sicht dient Personalabbau dazu, personelle Über- bzw. Fehlkapazitäten zu beseitigen, um ein gestörtes Gleichgewicht zwischen qualitativem und quantitativem Leistungsbedarf und Leistungsangebot seitens der Mitarbeiter wieder herzustellen" (Marr/Steiner 2003: 7).

„Aus betriebswirtschaftlicher Sicht steht das Potential und nicht die Person des betroffenen Arbeitnehmers im Vordergrund“ (ebd.: 7f.).

„Das Objekt eines Personalabbaus nach diesem Verständnis ist also eine Reduzierung betrieblich relevanter menschlicher Arbeitsleistung, nicht die Freisetzung spezieller Mitarbeiter“ (ebd.: 8 Anmerkung 16).

Aus marktgeprägter „betriebswirtschaftlicher Sicht“ geht es also nicht um den einzelnen Menschen. ,Downsizing' als bewusst gesteuerte ,Personalkapazitätsreduzierung' zielt stets auf Effizienzsteigerungen und nicht auf die Weiterbeschäftigung einzelner Mitarbeiterinnen oder Mitarbeiter (vgl. ebd.: 45).

Bei Dirk Kurbjuweit findet sich ein anschauliches Beispiel für diese rein ,betriebswirtschaftliche' Sicht der Dinge:

„Als George Kerschbaumer 24 Jahre alt war, bekam er seinen ersten Auftrag von McKinsey. Ein ländliches Maschinenbauunternehmen steckte in Schwierigkeiten. Die Leute, denen Kerschbaumer erklären sollte, wo es langgeht, waren doppelt so alt wie er. Von denen, sagte er, sei er ganz schön ,gechallenged' worden. Nach der Analyse empfahlen Kerschbaumer und zwei andere McKinseyMenschen, 20 bis 30 Prozent der Arbeitsplätze abzubauen. Sie meinten niemanden persönlich. Kerschbaumer gönnt jedem seinen Job. In den blauen Kladden stehen keine Namen, nur Graphiken, Pfeile, Zahlen. Wenn die Betroffenen erfahren, dass sie ihren Job verlieren, sind die McKinsey-Menschen längst in einem anderen Betrieb.

Herbert Henzler, lange Chef von McKinsey Deutschland, sagte mir, er sei ,nicht unfroh' über diese Distanz. ,Ich möchte das nicht entscheiden: Müller geht, Meier bleibt.' (...)

Das Image des McKinsey-Menschen ist das eines Jobkillers. Als McKinsey die Post AG untersucht hatte, empfahlen die Berater am Ende, dass $70.000 \mathrm{Ar}-$ beitsplätze abgebaut werden sollen, theoretisch alle Jobs einer kleineren Großstadt. Das war die wohl größte Zahl, die in einem Gutachten stand. Meist geht es um Hunderte, manchmal um Tausende Jobs. Zum Wort Jobkiller' sagt Kerschbaumer: Hätte das Landmaschinenunternehmen, das er beraten hat, nicht Arbeitsplätze abgebaut, wäre es wenig später kaputtgegangen. Insofern habe er Jobs gerettet, nicht vernichtet“" (Kurbjuweit 2004: 25f.).

Dass in einem Unternehmen, welches nicht durch systematischen Personalabbau geprägt ist, eine ähnliche Logik herrscht, veranschaulicht der amerikanische Wirtschafts-

5 Wie wenig bislang in der personalpolitischen betriebswirtschaftlichen Literatur ethische Fragestellungen eine Rolle spielten, verdeutlichen: Haueisen/Viertelhaus 1998: $719 \mathrm{f}$. 
ethiker Badaracco in einem Ratgeber für Führungskräfte. Badaracco beschreibt den Fall des 35-jährigen Peter Adario. Adario leitete die Marketingabteilung der Firma Sayer MircoWorld, die Computerprodukte vertrieb. Ihm war u. a. Lisa Walters unterstellt. In deren Team arbeitete seit erst vier Monaten die 37-jährige Kathryn McNeil. Sie war für die wichtigen IBM Kunden und ein Produktvolumen von monatlich 40 Millionen Dollars verantwortlich. Lisa Walters war ein Single Ende Zwanzig, sehr ehrgeizig und arbeitete mehr als andere. Adario war stolz darauf, dass er sich vor zwei Jahren für ihre Berufung zur Unterabteilungsleiterin eingesetzt hatte. Es war eine gute Entscheidung gewesen, die seine Abteilung gestärkt hatte.

„Walters wollte McNeil loswerden, weil sie ihr zu langsam war. McNeil war alleinerziehende Mutter eines sechsjährigen Kindes und bekam von ihrem ExEhemann keinerlei Unterhaltszahlungen. Walters glaubte, McNeils Pflichten zu Hause seien die Ursache ihrer Probleme im Job. Die Situation frustrierte Walters sehr, und ihr Geduldsfaden war am Zerreißen.

Adario fand, dass der Konflikt zwischen Walters und McNeil auch auf das Konto des hektischen Arbeitsklimas in der Firma ging. Das Computergeschäft war durch niedrige Gewinnmargen und schärfsten Konkurrenzkampf gekennzeichnet. (...) Unter diesem Druck waren Zehn- bis Zwölf-Stunden-Arbeitstage die Norm geworden, aber da die meisten Angestellten in Adarios Abteilung unverheiratete Männer und Frauen zwischen zwanzig und dreißig Jahren waren, führten die Überstunden bei ihnen nicht zu Konflikten zwischen Beruf und Familie.

Adario hatte Walters' Probleme nicht weiter zur Kenntnis genommen, bis er eines Morgens eine handgeschriebene Mitteilung auf seinem Stapel unerledigter Papiere fand. Es war Walters' zweites Memo in zwei Wochen. In beiden Memos klagte sie, dass McNeil nicht genug arbeitete, und forderte ihre Entlassung. Adario begriff: Von allein würde sich der Konflikt zwischen den beiden Frauen nicht lösen; er musste eingreifen. Und er hätte auch schon eingegriffen, schlügen nicht zwei Seelen in seiner Brust.

Einerseits leistete McNeil trotz einer 60-Stunden-Woche nicht genug. Alle anderen, auch Adario selbst, arbeiteten noch länger. (...) McNeil fuhr bei ihrer Bearbeitung der überaus wichtigen IBM-Kunden eindeutig Verspätungen ein, und ihr Verhältnis zu Walters hatte sich stark verschlechtert. Wenn Adario an all die Arbeit dachte, die die Abteilung erwartete, musste er Walters recht geben: Jawohl, er sollte einen Ersatz für McNeil finden und ihr kündigen.

Doch da war etwas, das Adario Bauchschmerzen machte. Hatte es nicht mehrfach geheißen, dass die Firma ein ,familienfreundliches' Betriebsklima förderte? Man hatte sogar eine Arbeitsgruppe eingesetzt, die untersuchen sollte, wie dies erreicht werden konnte. War da nicht der Fall Kathryn McNeil eine hervorragende Gelegenheit, endlich etwas Konkretes zu unternehmen? Adario sah sich nicht als Missionar oder Reformator, aber er war überzeugt, dass man besser arbeiten konnte, wenn mit dem restlichen Leben alles im Lot war.

Seine persönlichen Erfahrungen bestärkten Adario in dieser Überzeugung. Wie McNeil hatte er kaum Zeit für seine Familie. Seine Frau hatte nach der Geburt des zweiten Kindes und nachdem der zweite Arbeitgeber ihr starre Arbeitszeiten vorgegeben hatte, ihren Buchhalterberuf aufgeben müssen. Adarios Nachbar war vor drei Jahren bei Umstrukturierungen in seiner Firma entlassen worden, 
und obwohl er bald eine neue Stelle fand, war sein Selbstvertrauen schwer lädiert. Und überhaupt: Adario fand es nicht richtig, jemanden zu feuern, der wirklich hart arbeitete - noch dazu eine alleinerziehende Mutter (...) “ (Bardaracco 1998: 29-31).

„Adario schickte E-Mail-Nachrichten an Walters und McNeil und verließ dann sein Büro, um eine Präsentation auf einer Fachmesse zu geben. Er hatte ein gutes Gefühl. Er machte es sich nicht so leicht wie der Chef, der seinen Nachbarn gefeuert hatte; er versuchte, McNeils Job zu retten. Und er trat für das ein, woran er glaubte - zum Beispiel, dass man Menschen anständig zu behandeln hatte. Er tat sogar etwas für McNeils Sohn. Er freute sich schon darauf, seiner Frau berichten zu können, wie er das Problem angegangen war. (...) Doch dann kam der große Knall: Als er in sein Büro zurückkam, hörte er als erstes, dass McNeil gekündigt worden war.

Was war geschehen? Walters hatte das Problem mit mehreren anderen Managern besprochen, darunter ein Bereichsleiter, der ihr angeboten hatte ihr zu helfen, McNeil zu feuern. Als McNeil am frühen Nachmittag in ihr Büro kam, warteten Walters und der Bereichsleiter auf sie. Sie überreichten ihr ein Schreiben, in dem es hieß, dass ihre, Zusammenarbeit mit der Abteilungsleitung nicht zufriedenstellend' war. Sie wiesen sie an, für den Rest des Nachmittages ihre Nachfolgerin einzuarbeiten. (...) Danach sollte sie ihren letzten Gehaltsscheck plus zwei Wochen Abfindung abholen, ihre Siebensachen packen - und gehen“ (ebd.: 122f.).

Adario ist mit den Gepflogenheiten seiner Firma wohl vertraut. Es ist ein sehr leistungsorientiertes Unternehmen, welches sich in einem harten Konkurrenzkampf zur Erwirtschaftung von Gewinnen befindet. Und doch versucht Adario in dem Fall von McNeil familienorientierte Werte über die ökonomische Rationalität zu stellen. McNeil leistet eindeutig weniger als andere. Sie ist nicht ein Mitglied seiner Familie, die er zu schützen hat, sondern eine Mitarbeiterin in seiner Marketingabteilung, diese wiederum ist Teil der Firma Sayer MicroWorld, in der es noch andere Hierarchieebenen gibt, deren Orientierungswert ebenfalls sachlich zu erbringende Leistung und nicht der Erhalt persönlicher Beziehung ist. Adario hatte dies aus dem Blick verloren; er machte sich nicht bewusst, welche Leitwerte in seiner Firma dominant waren.

„Sein nobler Versuch, den Wertekurs seiner Firma familienfreundlicher zu prägen, war gründlich gescheitert“" (ebd.: 124).

In der Familie ist es unmoralisch, ein Mitglied dieser Familie zu entlassen, wenn es weniger leistet als ein anderes Familienmitglied. Familien sind gerade davon geprägt, leistungsschwache Personen nicht auszustoßen; sei es das allein nicht überlebensfähige Baby, sei es eine alte pflegebedürftige Mutter (vgl. Simon 2005a: 20). Eine Familie kann nicht Konkurs gehen, ganz gleich, was passiert; sie zerbricht auch nicht daran, wenn keine monetären Einnahmen mehr eingehen (vgl. ebd.: 27). Dadurch unterscheiden sie sich gründlich von einem primär marktwirtschaftlich ausgerichteten Unternehmen. In solch einem Unternehmen gilt allerdings - wie das Beispiel von Adarios Firma anschaulich macht - eine andere Moral. Wer nicht genug leistet, soll durch eine andere leistungsstärkere Person ersetzt werden. Menschen sind lediglich Mittel zum Zweck, insofern sind sie immer auch austausch- bzw. kündbar, wenn sie diesem Zweck nicht mehr dienen (vgl. ebd.: 21). 
Ein ganz zentrales Unterscheidungsmerkmal von Familienmoral und Marktmoral ist die unterschiedliche Gewichtung des Beziehungs- und des Sachaspektes. Während die Moral der Familie den Wert der dauerhaften Beziehung hoch hält, spielt er in der Moral des Unternehmens eine der Leistung untergeordnete Rolle.

\begin{abstract}
„Als Sozialsystem können Familie und Betrieb unterschiedlicher gar nicht sein. Für die Familie gibt es nichts Wichtigeres als die Person. Ihre Präsenz auch dann (vielleicht sogar gerade dann), wenn sie abwesend ist, ist wichtiger als das, was sie kann oder nicht kann, so sehr der heutige Arbeitsmarkt dazu zwingt, Bewertungen von Kompetenzen mitzuführen. Ihre Geschichte, in allen ihren biographischen Details, wird gewusst, erzählt und zelebriert, und dies auch und gerade dann, wenn sie zu äußerst kantigen Profilen führt, denn gerade daran erweist sich ja die Person als Person (und Persönlichkeit). Im Betrieb dagegen, so sehr Personen auch hier dazu neigen, Personen wahrzunehmen, zählt an erster Stelle die Sache. Personen kommen tendenziell nur als Beitrag zur Sache vor und fallen gerade dann besonders auf, wenn dieser Beitrag überdurchschnittlich ist oder sehr zu wünschen übrig lässt" (Baecker 2003b: 126f.).
\end{abstract}

Im Fall Kathryn McNeil war es letzteres. Dies führte zu ihrer Entlassung. Obwohl ihr Chef Adario durchaus die Person Kathryn McNeil in den Mittelpunkt seiner Überlegungen stellt, führt letztlich das Argument, dass die Leistung nicht ausreicht, zur Entlassung von Kathryn McNeil. Marktmoral betrachtet sachliche Leistung und tauscht Personen aus, wenn dies im Sinne der Leistungssteigerung möglich ist. Familienmoral würde versuchen, die Person zu fördern, damit sie ihre Leistung steigern kann. Letzteres wollte Adario versuchen, es hätte beinahe seine eigene Stelle gekostet, da er den marktmoralischen Einfluss in seiner Firma nicht richtig eingeschätzt hatte.

Natürlich ist das eine idealtypische Gegenüberstellung von der Moral der Familie und der Moral des Marktes. In der Realität wird es marktorientiert miteinander umgehende Familien ebenso geben wie familienorientierte Unternehmen. Und doch ist grundlegend festzuhalten, dass es ganz unterschiedliche Handlungs- und Wertorientierungen in Familien und marktorientierten Unternehmen gibt. Sicherlich wäre auch denkbar gewesen, dass der Boss von Adario soeben Vater geworden ist und sich ähnlich wie Adario nicht dafür entschließen kann, der Kündigungsbitte von Walters zu entsprechen. Dann wäre die Geschichte anders ausgegangen. Adario hätte familienorientierte Werte ins Feld führen können, um die Kündigung von $\mathrm{NcNeil} \mathrm{zu} \mathrm{verhindern.} \mathrm{Es} \mathrm{wäre}$ aber das gewesen, was das Wort, verhindert' ausdrückt, es hätte nicht die grundlegende Leistungsorientierung des Unternehmens verändern können. Dies hat in gewinnorientierten Unternehmen wie Sayer MircoWorld immer Priorität vor einer Familienorientierung. Wenn McNeil z. B. noch weniger arbeiten könnte, weil ihr Kind krank würde oder die Betreuung nicht mehr gesichert gewesen wäre; oder wenn die Fusionskrise bei Sayer MicroWorld für den Bestand des Unternehmens noch bedrohlicher geworden wäre und die Mitarbeitenden noch mehr hätten arbeiten müssen, um ihre eigenen Stellen zu sichern, wäre Adarios Entscheidung aller Wahrscheinlichkeit anders ausgefallen - oder wenn nicht, dann hätte er seinen Job verloren und Walters oder eine andere leistungsorientierte Person hätte seinen Marketingabteilungsleiterposten eingenommen. Undenkbar ist allerdings, dass Sayer MicroWorld McNeil und alle anderen Mitarbeiter mit persönlichen Leistungseinschränkungen behalten hätte. Wenn das Unternehmen Sayer MicroWorld sich dennoch so verhalten würde, wäre der si- 
chere Konkurs in einer marktwirtschaftlichen Umwelt vorgegeben, die sich nicht so verhält (vgl. Steinmann/Löhr 1994: 213-225). Adario kann die herrschende Unternehmensmoral nicht außer Kraft setzen und Sayer MicroWorld kann nicht die herrschende marktwirtschaftliche Moral außer Kraft setzen. Adario würde irgendwann, wenn er zu sehr familienorientiert entscheidet, seine eigene Stelle verlieren und Sayer MicroWorld, würde, wenn es zu viele Entscheider zulässt, die sich wie Adario verhalten, selbst Konkurs gehen (vgl. Simon 2005b: 61f.). Eine Kündigung Adarios würde aber umso deutlicher die herrschende Marktmoral bestätigen und andere Chefs in Zukunft von eben solchem Verhalten abhalten. Ebenso würde ein Konkurs von Sayer MicroWorld andere Unternehmen darin bestätigen, sich primär auf Leistung zu konzentrieren und sich, - wenn irgend möglich - von leistungsschwächeren Mitarbeitenden rechtzeitig zu trennen (vgl. Baecker/Kluge 2003: 113).

Marktwirtschaft funktioniert mitunter nach kurzfristiger Logik. „Der freie Kapitalmarkt gehorcht einer anderen Rationalität als eine Familie. Er ist meist an kurzfristiger Rentabilität interessiert, eine Familie hingegen an langfristiger Zukunftssicherung" (Simon 2005c: 370). Dies lässt sich auch am dargestellten Beispiel der Sayer MicroWorld Geschichte klar machen. Wenn eine prinzipiell leistungsstarke alleinerziehende Frau wie McNeil keine Chance hat, ihr Kind durch ihre Arbeit zu ernähren, weil der innerbetriebliche Konkurrenzkampf sie selektiert, dann entsteht dadurch langfristig die Gefahr fehlenden Nachwuchses; sowohl weil die Geburten zurückgehen, als auch weil ihm wenig psychosoziale Betreuungszeit gewidmet werden kann (vgl. Piorkowsky 2001: 14). Die auf Leistung ausgerichtete Moral der Marktwirtschaft selbst kann die für den Bestand der Gesellschaft notwendigen biosozialen Aufgaben der Nachwuchssicherung nicht gewährleisten. Und ebenso wenig kann die Moral der Marktwirtschaft die Regulation des Gefühlslebens übernehmen (vgl. Piorkowsky 1996: 53).

In der Marktwirtschaft herrscht eine andere Moral als in der Familie. Hier ist es eine Moral der Leistung, oder in der Sprache einer heutigen Ökonomik ausgedrückt: eine Vorteils-/Nachteils-Grammatik (vgl. Homann/Suchanek 2000: 440). Danach wird eingestellt, wer gebraucht wird und entlassen, wer überflüssig ist, d. h. wer überzählig ist oder wessen Leistung nicht mehr ausreicht (vgl. Marr/Steiner 2003: 7). Allerdings gilt es in der Welt der Marktwirtschaft auch nicht als unmoralisch, wenn eine Mitarbeiterin wie McNeil von sich aus ihren Arbeitsvertrag mit ihrer Firma gekündigt hätte, weil es für sie bestimmte Vorteile gebracht hätte, sei es, dass sie bei gleichem Lohn weniger hätte arbeiten müssen, sei es, dass sie sich ganz für die Erziehung ihres Kindes entschieden hätte, sei es, dass sie auf überraschend neue Weise eine gute Betreuungsmöglichkeit für ihr Kind gefunden hätte und deshalb durch einen Stellenwechsel sogar einen Karrieresprung hätte machen können. In einer Familie wäre das unmoralisch. Man lässt die Familie nicht im Stich (vgl. Simon 2005c: 23).

Wie kam es dazu, dass es diese beiden Moralvorstellungen gibt, die Peter Adario als so widersprüchlich erlebt und die ihn beinahe seinen Job gekostet hätten? Dazu bietet sich ein Blick in die Geschichte der ökonomischen Leitideen an. 


\section{Der Wandel der Ökonomie: Von der Hauswirtschaft zur Marktwirtschaft}

„Ökonomie, dieses Wort kommt von oikos, Haus und nomos, Gesetz. Gewöhnlich meint es die weise und legitime Regierung des Hauses, zum Zwecke des Gemeinwohls der ganzen Familie.

Die Bedeutung dieses Begriffs wurde in der Folgezeit ausgedehnt auf die Regierung der großen Familie, die der Staat ist. Um diese beiden Bedeutungen zu unterscheiden, nennt man sie im letzten Fall allgemeine Ökonomie oder politische Ökonomie und im anderen Fall Hauswirtschaft oder besondere Wirtschaft" (Rousseau 1755: 337).

Dieser 1755 von Jean Jacques Rousseau hergestellte Zusammenhang von Hauswirtschaft und Betriebswirtschaft, verliert kurz darauf seine hier so selbstverständlich erscheinende Verbindung. Ökonomie so wie sie Jean Jacques Rousseau definiert, schaut auf eine über zwei Jahrtausende währende Tradition zurück, die im wesentlichen durch Aristoteles geprägt worden ist (vgl. Burkhardt 1992: 511-513). ${ }^{6}$ Sie ist gerade dadurch gekennzeichnet, dass das, was heute im Mittelpunkt des wirtschaftswissenschaftlichen Interesses steht, keine Bedeutung für sie hat. In der aristotelischen Tradition steht nicht der Markt mit seiner an Tausch, Leistung, Wachstum und Gewinn orientierten Handlungsweise im Mittelpunkt ökonomischer Betrachtung, sondern eine - zunächst überwiegend landwirtschaftlich ausgerichtete - Hauswirtschaft, deren ökonomische Leitwerte sich am Erhalt des Bestehenden ausrichteten (vgl. Meran 2000: 23) und die von ihrer Einbettung in die polis her gedacht und konzipiert wurde (vgl. Adloff 2005: 18; Spahn 1992: 517). ${ }^{7}$ Der Ökonom soll nicht bestrebt sein, Gewinne um ihrer selbst zu machen, sondern er wirtschaftet zum Zweck eines tugendhaften menschlichen Miteinanders:

„Nun ist aber offenbar, dass die Tätigkeit der Hausverwaltung (oikonomia) ihre Bestrebungen in höherem Grade auf die Menschen als auf leblosen Besitz richtet und mehr auf die Tugend der Menschen als auf die Anhäufung von Besitztümern, die man Reichtum nennt, (...)“ (Aristoteles 4. Jh. v. Chr.: 71f.).

In dieser Tradition stehend, lässt es Leon Battista Alberti knapp 2000 Jahre später seinen Oeconomicus Gianozzo so formulieren:

„Von Natur aus macht mir Liebe und Pflichterfüllung die Familie zum teuersten aller Güter; aber um die Familie zu erhalten, erstrebt man Besitz; (...) “ (Alberti 1437-41: 238).

$6 \quad$ Vgl. dazu auch Hoffmann 1959: 207f. und Meyer 1998: 52. Spahn weist darauf hin, dass die Ökonomie des Aristoteles sich an antike Vorgänger anschließt, die schon eine Abwehrhaltung gegenüber einer sich rasch entwickelnden Marktdynamik hatten (vgl. Spahn 1992: 521).

7 „Man rechnet nicht damit, dass der Hausvater (...) jemals mehr erwirbt, als zur Sicherung eines standesgemäßes Unterhaltes seiner selbst, seiner Frau und seiner Kinder nötig ist." (Hoffmann 1959: 188). 
1776 holt Adam Smith das von Aristoteles verbannte Gewinnstreben zurück in die Ökonomie (vgl. dazu auch Ulrich 2001: 173) ${ }^{8}$ Er interessiert sich intensiv für die Austauchprozesse, die außerhalb des Hauses auf dem Markt geschehen. Erwerb, Gewinn und Wachstum werden in den Mittelpunkt der Betrachtung gestellt und von ihrer bislang gesellschaftlichen Ächtung befreit (vgl. Richarz 2001: 24). Die ,invisible hand", der Markt, wird als moralische Instanz eingeführt. ${ }^{9}$ Damit ist die durch Aristoteles in der Antike festgeschriebene Abwertung des Strebens nach Gewinn wissenschaftlich überwunden (vgl. Brunner 1968: 105). Und die aristotelische Tradition den Zweck des Wirtschaftens im tugendhaften Erhalt der Familie zu sehen, verliert sich. Das Gewinnmaximierungsprinzip wird zum Selbstzweck (vgl. Weber 1920: 44).

Es kommt zu einer folgenreichen Werteumkehr. Die Ökonomie der Hauswirtschaft verwandelt sich in eine Ökonomie der Marktwirtschaft. War in der hauswirtschaftlich geprägten Ökonomie der Tauschhandel geringgeschätzt (vgl. Gurjewitsch 2004: 268311), so ist es in der marktwirtschaftlich geprägten Ökonomie die Arbeit im Haus: Die nicht gewinnorientierte - nicht auf Austausch angelegte - Produktion und Dienstleistung (vgl. Hausen 1976: 319)..$^{10}$ Die auf Erhalt des Bestehenden gerichtete Hauswirtschaft verliert ihren Wert und entschwindet mehr und mehr aus dem ökonomischen Blickfeld der sich im 19. und 20. Jahrhundert schnell entwickelnden neuen Wirtschaftswissenschaft (vgl. Richarz 2001: 25; Brunner 1968: 119). Von dieser Geringschätzung ist auch die Betriebswirtschaftslehre betroffen, auf die bis weit ins 20. Jahrhundert hinein herabgeschaut wurde (vgl. Wöhe 1984: 65-68). Die Betriebswirtschaftslehre konstituiert sich nicht als neue, individualistische Hauswirtschaftslehre, sondern als eine das Gewinnmaximierungsprinzip aus der Volkswirtschaftslehre übernehmende Wissenschaft. ${ }^{11}$ Damit ist weder das in der aristotelischen Tradition stehende Maßhalten, noch die Orientierung an einem menschlich-tugendhaften Miteinander zum Zweck der Betriebwirtschaftslehre geworden. Betriebswirtschaft ist - anders als in der früheren hauswirtschaftlichen Ökonomie - von einem primär sachlichen Zweck (Gewinn) bestimmt. Personen werden somit Mittel zum Zweck. ${ }^{12}$

Das volkswirtschaftliche Gewinnprinzip, welches besagt, dass der Wohlstand aller dadurch gesteigert werde, wenn jeder Einzelne bestrebt sei, seinen individuellen Nutzen zu mehren, wird auch zum handlungsleitenden moralischen Prinzip in den Unternehmen. Familienorientierte Werte gibt es nach wie vor. Doch werden sie nun von

8 So gelingt es z. B. den Niederländern in ihrem goldenen Zeitalter des Handels noch nicht, eine neue Wirtschaftstheorie zu begründen, die in der Lage gewesen wäre, das Gewinnprinzip moralisch zu legitimieren (vgl. Schama 1988: 315-401).

9 Die moralphilosophische Ergänzung durch das Sympathieprinzip wurde in der Wirtschaftswissenschaft kaum rezipiert (vgl. Ulrich 2001: 63-66).

$10 \quad$ Vgl. Freudenthal 1986; Frevert 1995: 144-165; Niehuss 1999: 46; Sieder 1987: 244; Wunder 1992.

11 Vgl. Baecker 2003a: 9-11; Wöhe 1984: 6, 70; Wöhe 2005: 65; Heinen 1991: 16; Ulrich 2001: 413416.

12 Neben der Betriebswirtschaftslehre entstand die Managementlehre (vgl. Staehle 1994: 71-74) und innerhalb der Betriebswirtschaftslehre die Personalwirtschaftslehre. Die Personalwirtschaftslehre ist ebenso wie die Betriebwirtschaftslehre dominiert vom Gewinnmaximierungsprinzip. Und der Managementlehre ist es bislang noch nicht gelungen, die Unternehmensführung auf einem sicheren ethischen Fundament aufzubauen. (vgl. ebd.: 586-594). 
außen an das Unternehmen herangetragen, wie am Beispiel von Peter Adario zu sehen ist. In den Unternehmen wird letztlich nach dem Gewinnmaximierungsprinzip gehandelt. Etwa wenn es darum geht, Personal abzubauen, dessen Leistung wie im Fall von Kathryn McNeill als nicht ausreichend bewertet wird, oder wenn es darum geht, ganze Unternehmen umzustrukturieren, wie in den anfangs zitierten Beispielen aus der gewinn- und leistungsorientierten McKinseywelt. Das Argument dabei ist, dass der Markt nur die Gewinner überleben lässt und Unternehmen, die nicht konsequent gewinnorientiert handeln, vom Markt verschwinden werden. Dass das Gewinnprinzip solche Macht bekommen hat, lässt sich vielleicht aber auch durch den Verlust der maßhaltenden Moral der aristotelischen Ökonomie innerhalb der Unternehmen erklären. Denn wenn das Gewinnstreben der wesentliche Unternehmenszweck aller am Markt wirtschaftenden Unternehmen ist, dann muss man sich nicht wundern, wenn der Markt lediglich vom Gewinnmaximierungsprinzip dominiert ist und dies wiederum selbstverstärkende Rückwirkungen in die Unternehmen hat (vgl. Baecker 1999: 179; Beschorner 2004: 156). Gewinn fordert noch mehr Gewinn; Leistung fordert immer bessere Leistung. Denkbar wäre es durchaus, dass ausgehend von den Unternehmen eine andere Moral des Marktes entsteht. Eine Moral, in der Gewinn- und Leistungsorientierung nach wie vor erlaubt und nicht begrenzt, sondern ergänzt wird von einer lebensdienlichen Ethik (vgl. Ulrich 2001: 427-437), die auch dem Inne- und dem Maßhalten (vgl. ebd.: 226) Raum gibt. Oder eine Marktmoral, die ein ökonomisches Prinzip neben einem kulturellen Prinzip kennt, beide mit jeweils unterschiedlichen Geschwindigkeiten und beide aufeinander bezogen (vgl. Röttgers 2003: 12). Eine Moral, die weiterhin die Ökonomisierung der Kultur zulässt; die aber auch zulässt, dass nicht nur der Gewinner überlebt, sondern auch der Verlierer wieder Gewinner werden kann, weil Umwege, Reflexionen, Moratorien, Pausen, Verhandlungen, Rituale, u. a. durch eine „Kultivierung der Ökonomie“ (Röttgers 2004: 127) möglich werden. Kurzfristige gewinnorientierte Sachentscheidungen in Verbindung mit langfristigen Personalentscheidungen würden denkbar, ohne dabei in vormoderne Machtrelationen zurückfallen zu müssen (vgl. Simon 2005c: 370-372).

Denn eine Gesellschaft, in der nur Lisa Walters eine Chance hat, sich individuell zu entwickeln, kann ethisch nicht wünschbar sein (vgl. Ulrich 2002: 38). Auch Kathryn McNeil darf nicht nur als Mittel zum Zweck gesehen werden. Eine Gesellschaft, die es Sayer MicroWorld ermöglichen würde, entweder McNeil weiter zu beschäftigen, weil die Unternehmen familienorientierter arbeiten, oder aber die McNeil weitere individuelle Entwicklungschancen bieten könnte, ohne durch eine Kündigung existenziell gefährdet zu sein, würde sich mit Kants Begriff von Würde in Einklang bringen lassen (Ulrich 2001: 71f., 238).

„Der Mensch aber ist keine Sache, mithin nicht etwas, das bloß als Mittel gebraucht werden kann, sondern muß bei allen seinen Handlungen jederzeit als Zweck an sich selbst betrachtet werden" (Kant 1965: 52).

Wenn Unternehmen Personal abbauen, dann fragt sich, wer die ggf. für immer ausgemusterten Menschen als dazugehörig und wertvoll aufnimmt (vgl. Sennett 2000: 180). Marktwirtschaft ist ein Selektionsmechanismus, der die Starken begünstigt (vgl. Samuelson 1981: 68). Die Moral der Leistung hilft nicht weiter, wenn jemand schwach wird. Wer in der Wirtschaft als überflüssig selektiert wird, der fällt - wenn er Glück 
hat - in ein soziales Netzwerk, sei es die eigene Familie, ${ }^{13}$ sei es ein staatlich garantiertes Netzwerk zurück. Familiäre und oder staatliche Netzwerke fangen einen als leistungsschwach vom Marktmechanismus bewerteten Menschen auf und können ihn nun nach eigener Beziehungsmoral nicht einfach schnell selbst wieder (wohin denn auch?) entlassen. Es sei denn dieser als relativ leistungsschwach von einem einzelnen Unternehmen bewertete Mensch findet woanders in einer neuen Relationswertbeziehung einen neuen wirtschaftlichen Marktwert. Die Unterstützung dieser individuell ausgerichteten Eigenständigkeit könnte Aufgabe und Zweck familiärer, staatlicher, unternehmerischer oder auch neu entstehender (vgl. Sennett 2007: 146f.) Beziehungsnetzwerke sein.

\section{Literaturverzeichnis}

Adloff, F. (2005): Zivilgesellschaft. Theorie und politische Praxis, Frankfurt a. M.: Campus.

Alberti, L. B. (1437-41/1986): Vom Hauswesen (Della Famiglia), München: dtv klassik.

Aristoteles (4. Jh. v. Chr./2003): Politik, Hamburg: rowohlts enzyklopädie.

Baecker, D. (1999): Die Form des Unternehmens, Frankfurt a. M.: Suhrkamp.

Baecker, D. (2003a): Einleitung, in: Ders. (Hrsg.): Organisation und Management. Aufsätze, Frankfurt a. M.: Suhrkamp, 9-17.

Baecker, D. (2003b): Tabus in Familienunternehmen, in: Ders. (Hrsg.): Organisation und Management. Aufsätze, Frankfurt a. M.: Suhrkamp, 123-133.

Baecker, D./ Kluge, A. (2003): Vom Nutzen ungelöster Probleme, Berlin: Merve.

Beschorner, Th. (2004): Unternehmensethiken. Eine theoretische Einführung, in: Beschorner, Th./ Schmidt, M. (Hrsg.): Integritäts- und Umweltmanagement in der Beratungspraxis, München und Mering: Hampp, 151-178.

Badaracco, J. L. (1998): Manager zwischen gut und richtig. So entscheiden Sie in schwierigen Führungssituationen, Landsberg/Lech: mvg.

Bertram, H. (2000): Die verborgenen familiären Beziehungen in Deutschland: Die multilokale Mehrgenerationenfamilie, in: Kohli, M./ Szydlik, M. (Hrsg.): Generationen in Familie und Gesellschaft, Opladen: Leske + Budrich, 97-121.

Brunner, O. (1968): Das „ganze Haus“ und die alteuropäische „Ökonomik“, in: Ders. (Hrsg.): Neue Wege der Verfassungs- und Sozialgeschichte, 2. Auflage, Göttingen: Vandenhoeck \& Ruprecht, 103-127.

Burkhardt, J. (1992): Artikel: Wirtschaft. I. Einleitung, in: Brunner, O./ Conze, W./ Koselleck, R. (Hrsg.): Geschichtliche Grundbegriffe. Historisches Lexikon zur politisch-sozialen Sprache in Deutschland, Band 7, Stuttgart: Klett-Cotta, 511-513.

Frevert, U. (1995): „Mann und Weib, und Weib und Mann“. Geschlechter-Differenzen in der Moderne, München: Beck.

Freudenthal, M. (1986): Gestaltwandel der städtischen, bürgerlichen und proletarischen Hauswirtschaft zwischen 1760 und 1910, hg. v. K. Rutschky, Frankfurt a. M.: Ullstein.

13 Vgl. zu der nach wie vor großen Bedeutung der emotionalen Beziehungsfunktion der Familie trotz Pluralisierung der Haushaltsformen Piorkowsky 1996: 57f. und dies unterstreichend sowie die heute weit verbreitete Netzwerkstruktur von Familien herausarbeitend Bertram 2000: 97-121. 
Gurjewitsch, A. J. (2004): Der Kaufmann, in: Le Goff, J. (Hrsg.): Der Mensch des Mittelalters, Essen: Magnus, 268-311.

Haueisen, G. K./ Viertelhaus, M. (1998): Betriebliche Sozialpolitik und Personalmanagement als Problemfelder der Unternehmensethik, in: Clermont, A./ Schmeisser, W. (Hrsg.): Betriebliche Personalpolitik, München: Vahlen, 719-741.

Hausen, K. (1976): Die Polarisierung der „Geschlechtscharaktere“ - Eine Spiegelung der Dissoziation von Erwerbs- und Familienleben, in: Conze, W. (Hrsg,): Sozialgeschichte der Familie in der Neuzeit Europas, Stuttgart: Klett.

Heinen, E. (1991): Industriebetriebswirtschaftslehre. Entscheidungen im Industriebetrieb, 9. vollständig neu überarbeitete und erweiterte Auflage Wiesbaden: Gabler.

Hoffmann, J. (1959): Die „Hausväterliteratur“ und die „Predigten über den christlichen Hausstand“. Lehre vom Hause und Bildung für das häusliche Leben im 16., 17. und 18. Jhdt., Berlin: Beltz.

Hollstein, B. (2005): Reziprozität in familialen Generationenbeziehungen, in: Adloff, F./ Mau, St. (Hrsg.): Vom Geben und Nehmen. Zur Soziologie der Reziprozität, Frankfurt/New York: Campus, 187-209.

Holtfrerich, C.-L. (2007): Wo sind die Jobs? Eine Streitschrift für mehr Arbeit, München: Deutsche Verlags-Anstalt.

Homann, K./ Suchanek, A. (2000): Ökonomik: Eine Einführung, Tübingen: Mohr Siebeck.

Kant, I. (1965): Grundlegung der Metaphysik der Sitten, hg. v. K. Vorländer, Nachdruck 3. Auflage Hamburg: Meiner.

Kurbjuweit, D. (2004): Unser effizientes Leben. Die Diktatur der Ökonomie und ihre Folgen, Hamburg: Rowohlt.

Marr, R./ Steiner, K. (2003): Personalabbau in deutschen Unternehmen. Empirische Ergebnisse zu Ursachen, Instrumenten und Folgewirkungen, Wiesbaden: Deutscher Universitätsverlag.

Meran, J. (2000): Wirtschaftsphilosophie. Kurseinheit 1: Wirtschaftsethik, 2. Auflage, FernUniversität Hagen.

Meyer, U. (1998): Soziales Handeln im Zeichen des ,Hauses'. Zur Ökonomik der Spätantike und im frühen Mittelalter, Göttingen: Vandenhoeck \& Ruprecht.

Middelhoff, Th. (2004): Vom Familienunternehmen zur börsennotierten Aktiengesellschaft, in: Brink, A./ Karitzki, O. (Hrsg.): Unternehmensethik in turbulenten Zeiten. Wirtschaftsführer über Ethik im Management, Bern u. a.: Haupt, 355-368.

Niehuss, M. (2004): Die Hausfrau, in: Frevert, U./ Haupt, H.-G. (Hrsg.): Der Mensch des 20. Jahrhunderts, Essen: Magnus, 45-65.

Pieper, A. (2001): Einführung in die philosophische Ethik (für die Wirtschaftsphilosophie bearbeitet) Kurseinheit 1: Gegenstand und Aufgabe der Ethik, FernUniversität Hagen.

Piorkowsky, M.-B. (1996): Haushaltsökonomie, in: Kutsch, Th./ Piorkowsky, M.-B./ Schätzke, M. (Hrsg,): Einführung in die Haushaltswissenschaft. Haushaltsökonomie, Haushaltssoziologie, Haushaltstechnik, Stuttgart: UTB, 5-184.

Piorkowsky, M.-B. (2001): Neue Hauswirtschaft. Ein Ansatz zur Neuorientierung der familienund haushaltsbezogenen Bildung am Anfang des 21. Jahrhunderts, in: http:// www.neuehauswirtschaft.de.

Richary, I. (2001): Der Haushalt in Wissenschaft und Bildung. Herausforderungen in sich wandelnder Welt, Hohengehren: Schneider.

Röttgers, K. (2003): Langsamkeit und Kultur, in: ftp://ftp.fernuni-hagen.de/pub/fachb/esgw /prph/preprints/la-ku.doc. 
Röttgers, K. (2004): Wirtschaftsphilosophie - Die erweiterte Perspektive, in: Zeitschrift für Wirtschafts- und Unternehmensethik Jg. 5/Heft 2, 114-133.

Rousseau, J. J. (1755/1966): Artikel „Ökonomie“, in: Encyclopédie ou Dictionnaire Raisonné des Sciences des Arts et des Métiers (Nouvelle Impression en facsimilé de la première édition de 1751 - 1780 Volume 5) Stuttgart, Bad Cannstatt: Frommann, 337.

Samuelson, P. A. (1981): Volkswirtschaftslehre. Eine Einführung, Band 1, Köln: Bund.

Schama, S. (1988): Überfluß und schöner Schein. Zur Kultur der Niederlande im Goldenen Zeitalter, München: Kindler.

Sennett, R. (2000): Der flexible Mensch. Die Kultur des neuen Kapitalismus, Berlin: Siedler.

Sennett, R. (2007): Die Kultur des neuen Kapitalismus, Berlin: Berliner Taschenbuch Verlag.

Sieder, R. (1987): Sozialgeschichte der Familie, Frankfurt a. M.: Suhrkamp.

Simon, F. B. (2005a): Familien und Unternehmen ... Überlegungen zu Unterschieden, Gemeinsamkeiten und den Folgen, in: Ders. (Hrsg.): Die Familie des Familienunternehmens. Ein System zwischen Gefühl und Geschäft, 2. Auflage, Heidelberg: Carl-Auer, 17-34.

Simon, F. B. (2005b): Gerechtigkeit, Richtigkeit und Rechtmäßigkeit ... Entscheidungsfindung in Familienunternehmen, in: Ders. (Hrsg.): Die Familie des Familienunternehmens. Ein System zwischen Gefühl und Geschäft, 2. Auflage, Heidelberg: Carl-Auer, 55-71.

Simon, F. B. (2005c): Nachbemerkung. Die Entkopplung von Familie und Unternehmen, in: ders. (Hrsg.): Die Familie des Familienunternehmens. Ein System zwischen Gefühl und Geschäft, 2. Auflage, Heidelberg: Carl-Auer, 367-372.

Smith, A. (1776): Reichtum der Nationen, Kirchheim: Oldenbourg.

Spahn, P. (1992): Artikel: Wirtschaft. II. Antike, in: Brunner, O./ Conze, W./ Koselleck, R. (Hrsg.): Geschichtliche Grundbegriffe. Historisches Lexikon zur politisch-sozialen Sprache in Deutschland, Band 7, Stuttgart: Klett-Cotta, 513-526.

Spree, R. (2003): Concerns about Globalisation - Then and Now, in: Münchener Wirtschaftswissenschaftliche Beiträge, 1-30, www.epub.ub.uni-muenchen.de/44 (abgerufen am 09.12.2007).

Staeble, W. H. (1994): Management. Eine verhaltenswissenschaftliche Perspektive, München: Vahlen.

Stierlin, H. (1997): Verrechnungsnotstände: Über Gerechtigkeit in sich wandelnde Beziehungen, in: Familiendynamik. Zeitschrift für systemorientierte Praxis und Forschung Jg. 22/Heft 2, 136-155.

Steinmann, H./ Löhr, A. (1994): Grundlagen der Unternehmensethik, 2. überarbeitete und erweiterte Auflage, Stuttgart: Schäffer-Poeschel.

Ulrich, P. (2001): Integrative Wirtschaftsethik. Grundlagen einer lebensdienlichen Ökonomie, 3. revidierte Auflage Bern u. a.: Paul Haupt.

Ulrich, P. (2002): Der entzauberte Markt. Eine wirtschaftethische Orientierung, Freiburg: Herder.

Weber, M. (1920/1984): Die protestantische Ethik, hg. v. J. Winckelmann, Gütersloh: GTB Siebenstern.

Wöhe, G. (1984): Einführung in die Allgemeine Betriebswirtschaftslehre, München: Vahlen.

Wöhe, G. (2005): Einführung in die Allgemeine Betriebswirtschaftslehre, München: Vahlen.

Wunder, H. (1992): „Er ist die Sonn’ sie ist der Mond“. Frauen in der Frühen Neuzeit, München: Beck. 